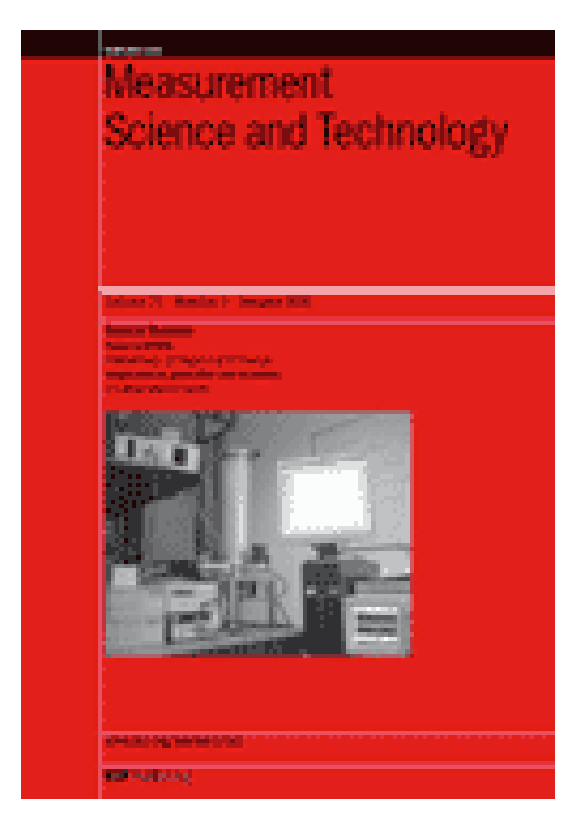

Title: "Automatic measurement of field-dependent elastic modulus and damping by laser Doppler vibrometry"

Authors: Morales A.L., Nieto A.J., Chicharro, J.M., Pintado P.

MEASUREMENT SCIENCE \& TECHNOLOGY, 19, pp. 125702-125713 (2008)

Keywords:

Magnetoelasticity, elastic modulus, damping, nickel

DOI: 10.1088/0957-0233/19/12/125702 


\title{
Automatic measurement of field-dependent elastic modulus and damping by laser Doppler vibrometry
}

\author{
AL MORALES, AJ NIETO, JM CHICHARRO and P \\ PINTADO \\ Área de Ingeniería Mecánica \\ ETSI Industriales (Universidad de Castilla - La Mancha) \\ Edificio Politécnico, Avda. Camilo José Cela s/n, 13071, Ciudad Real (Spain) \\ E-mail: AngelLuis.Morales@uclm.es
}

\begin{abstract}
A method for characterizing the magnetoelastic dependence of both Young's modulus and damping on the magnetic field is presented. It is based on laser Doppler vibrometry and free longitudinal vibration in soft ferromagnetic rods and wires, and offers a broad range of improved features including accuracy, lack of interaction with the sample, speed of measurement, full automation, high resolution, and the possibility of stress-dependence studies. All these allow samples to be perfectly characterized in the full magnetic field range, estimating the behaviour of the specimen as different magnetization curves are followed and discovering critical points that had been overlooked in previous works. As an example, the magnetoelastic characterization of nickel rods is described, and excellent results are obtained which are consistent with the hysteresis loop of nickel and the theory of magnetic domains in ferromagnetic materials.
\end{abstract}

PACS numbers: $75.80 .+\mathrm{q}, 62.40 .+\mathrm{i}, 43.20 . \mathrm{Ks}$

Keywords: magnetoelasticity, elastic modulus, damping, laser Doppler vibrometry, nickel

Submitted to: Meas. Sci. Technol. 


\section{Introduction}

The elastic characterization of magnetic materials requires knowledge of their state of magnetization, since this influences the elastic behaviour of the solid [1]. The main objective of the present work was to develop an experimental set-up which is able to improve the characterization of important magnetoelastic effects relative to existing techniques. Specifically, the effects studied are the dependence of both the elastic modulus and damping on the applied magnetic field, i.e., the so-called $\Delta E$-effect and magnetomechanical damping ( $\Delta \Psi$-effect for symmetry in nomenclature), respectively.

When tension is applied to any ferromagnetic sample, two different types of deformation appear: elastic $\left(\varepsilon_{11}\right)$, fully described by Hooke's law, and magnetoelastic $\left(\varepsilon_{\mathrm{ml}}\right)$, an additional strain caused by the magnetic state of the sample and the constitution of its magnetic domains [1, 2]. Hence, the existence of an applied magnetic field causes the apparent Young's modulus to differ from its value in the demagnetized state. It is known that stress can also alter the magnetic domain structure but for a low stress level within the specimen such influence can be neglected. The Young's modulus for a specific applied magnetic field will be called $E_{\mathrm{H}}$, so the complete $\Delta E$-effect is described in terms of the ratio:

$$
\frac{\Delta E}{E}=\frac{E_{\mathrm{S}}-E_{\mathrm{D}}}{E_{\mathrm{D}}}=\frac{\varepsilon_{\mathrm{ml}}}{\varepsilon_{11}}
$$

with $E_{\mathrm{D}}$ and $E_{\mathrm{S}}$ being the demagnetized and saturated Young's moduli, respectively.

Also, when materials are set in vibration some of the elastic energy is converted into heat, which leads to the extinction of the oscillation. As in the case of Young's modulus, the damping of elastic waves in ferromagnetic materials behaves differently when the material's magnetic domains are altered. Nevertheless, unlike elastic modulus, stress-dependence of damping in ferromagnetic materials cannot be neglected especially in the demagnetized state $[2,3]$, so domain motion is caused by the existence of both magnetic and stress interaction. This effect, commonly known as magnetomechanical damping, is studied in terms of the specific damping capacity $\Psi$, and hence our use of the term $\Delta \Psi$-effect in the present work. Precisely, the specific damping capacity is defined as the ratio between the energy dissipated per cycle $\Delta W$ and the maximum energy of the system $W$, and can be also obtained from of the logarithmic decrement $\delta$ which is the natural logarithm of the ratio between the amplitude of two consecutive free oscillations $A_{i}$ and $A_{i+n}$ :

$$
\Psi=\frac{\Delta W}{W}=2 \delta=2 \ln \left(\frac{A_{i}}{A_{i+1}}\right)=2 \frac{1}{n} \ln \left(\frac{A_{i}}{A_{i+n}}\right)
$$

The specific damping capacity for a specific applied magnetic field and stress will be called $\Psi_{\mathrm{H}, \sigma}$. So, for symmetry in expressions, the $\Delta \Psi$-effect can be described in terms of the ratio:

$$
\frac{\Delta \Psi}{\Psi}=\frac{\Psi_{\mathrm{S}, \sigma}-\Psi_{\mathrm{D}, \sigma}}{\Psi_{\mathrm{D}, \sigma}}
$$


with $\Psi_{\mathrm{D}, \sigma}$ and $\Psi_{\mathrm{S}, \sigma}$ being the demagnetized and saturated specific damping capacity for a constant stress $\sigma$.

A summary of the methods used to measure these effects is given by Squire in [4]. For the $\Delta E$-effect measurement in particular, some of the techniques described are based on the use of electromagnetic sensors and actuators. Two examples are the resonance and antiresonance method [5] and the ultrasonic wave velocity technique [6], both of which, however, present certain drawbacks. The former is unable to estimate the Young's modulus over the whole range of applied field, and in the latter the influence of the electromagnetic actuator may modify the magnetic state of the sample [4]. Other methods involve the sample's vibration. An example is the vibrating reed technique [7], where the sample is excited by an alternating electric field, and the resulting vibration is measured via capacitive or optical detectors. Again, the exciting electric field may become a non-negligible source of error. A later work has developed a method based on the longitudinal free vibration of the specimen and the measurement technique of heterodyne-speckle interferometry [8]. This system not only ensures that the vibration can be measured while avoiding contact with the sample but also provides a stress pulse which does not influence the sample's magnetic state. With respect to the characterization of magnetomechanical damping or $\Delta \Psi$-effect, the different methods are distinguished by the technique they use to measure the damping [9]: measurement of the attenuation of a stress pulse travelling through the material, estimation of the bandwidth which appears in the resonance curve of forced vibration, or determination of the decay of the free vibration time-response. Especially important is the torsional pendulum method [4, 10], which is used to measure both internal friction and shear modulus, and the aforementioned technique of heterodyne-speckle interferometry, which has been successfully tested in $\Delta \Psi$-effect characterization simultaneously with measurement of the $\Delta E$-effect [11].

In magnetic materials, due to the previously mentioned reciprocal dependence between magnetic and mechanical properties [1], the stress applied to a material may have influence on the measurement of field-dependent $E$ and $\Psi$. So, some methods based on forced vibration are centred on studying this influence up to stresses near the fatigue limit $[3,12,13,14]$. In methods which use free vibrations, like the torsional pendulum method, it is very difficult to keep the stress amplitude uniform enough to avoid its influence on the results, but Atalay [10] shows one way to consider and minimize this effect.

In short, it seems at present that only the heterodyne-speckle interferometry technique can provide simultaneous and accurate measurements of both the $\Delta E$-effect and the $\Delta \Psi$-effect over the full range of applied magnetic fields and without interacting with the mechanical or magnetic state of the sample. Nevertheless, as in all the above experimental techniques, not only significant but even critical improvements are possible. In particular, the present work describes an approach to full automation of the experimental process, sharply reduced characterization times, high-resolution characterization curves without loss of information and a more careful treatment of the 
stress level interaction.

In order to attain these goals, new excitation and measurement techniques were developed. For the excitation, we designed a fast, automatic process of free longitudinal vibration which does not interact with the specimen while it oscillates, and allows characterization over the full range of magnetization. For the measurement system, we chose a recently improved instrumentation system based on laser Doppler vibrometry (LDV).

This choice of LDV merits further explanation [15]. The first LDV models showed limited sensitivity, low signal-to-noise ratio (SNR), and poor applicability to nondiffusive surfaces. But developments since the early 1990s have greatly enhanced their measurement capabilities with respect to traditional sensors such as accelerometers and strain gauges, and even other interferometric techniques such as electronic speckle pattern interferometry (ESPI). Specifically, they allow remote, non-intrusive, high spatial resolution measurements with short testing times and improved performance (high-frequency bandwidth, greater linearity, broad velocity range, and high resolution in displacement and velocity measurements). Furthermore, they are able to measure usually difficult surfaces such as those with tight radii of curvature, edges, or very small structures, and even under difficult working conditions such as on hot parts or through glass windows or fluids. LDV has been applied to many areas of engineering and science, from mechanical engineering to biomedicine, micro- and nano-electromechanical systems (MEMS and NEMS), artworks, archaeology, and, of course, the characterization of smart materials. In particular, some authors have recently developed experimental systems based on non-contact laser vibrometry for determining vibration and sound fields $[16,17]$, for detecting fatigue cracks [18] or for estimating mechanical properties of different materials $[19,20]$. In the specific field of magnetoelastic characterizations, measurements of Young's modulus and damping have been carried out successfully by Morales in [21].

The basic idea behind the improvement of the characterization of the magnetoelastic effects in ferromagnetic materials is to take advantage of the particular features of the specially designed automatic excitation system and the LDV-technology-based instrumentation.

\section{Theoretical background}

Before describing the experimental set-up and the data processing used to estimate the $\Delta E$-effect and $\Delta \Psi$-effect from the acquired signals, we shall give a brief overview of the theory of the damped longitudinal propagation of a stress wave through an elastic and ferromagnetic material, the details of which may be found in [1]. The stress acting on a section is given by the sum of three terms: an elastic term proportional to strain,

a dissipative viscous term proportional to the temporal variation of the strain, and a magnetoelastic term related to the applied magnetic field. The resulting wave equation 
governing propagation is $[1,11]$ :

$$
\frac{\partial^{2} u_{\mathrm{z}}}{\partial z^{2}}=\frac{\rho}{E_{\mathrm{H}}} \frac{\partial^{2} u_{\mathrm{z}}}{\partial t^{2}}-\frac{\eta}{E_{\mathrm{H}}} \frac{\partial^{3} u_{\mathrm{z}}}{\partial z^{2} \partial t}+\frac{\lambda_{\mathrm{s}}}{M_{\mathrm{s}}} \frac{\partial H_{\mathrm{z}}}{\partial z}
$$

where $\rho$ is the material density, $\eta$ the structural damping loss factor, $E_{\mathrm{H}}$ the Young's modulus for the magnetic field $H_{\mathrm{z}}, \lambda_{\mathrm{s}}$ and $M_{\mathrm{s}}$ the saturation magnetostriction and magnetization, respectively, $\chi$ the magnetic susceptibility, and $u_{\mathrm{z}}$ the longitudinal displacement. In this case the longitudinal variation of the magnetic field is neglected since the field is assumed to be uniform over the entire length of the ferromagnetic specimen. This simplifies the wave equation which, with separation of variables, has the solution [11]:

$$
u_{\mathrm{z}}(z, t)=[\mathrm{A} \cos (k z)+\mathrm{B} \sin (k z)]\left[\mathrm{C} \cos \left(\omega_{\mathrm{d}} t\right)+\mathrm{D} \sin \left(\omega_{\mathrm{d}} t\right)\right] \exp \left(-\frac{\gamma}{2} t\right)
$$

with $k$ being the wave number, $\omega_{\mathrm{d}}$ the damped longitudinal angular frequency, $\frac{\gamma}{2}$ the attenuation constant, and A, B, C, and D constants which depend on the initial and boundary conditions. The $j^{\text {th }}$ damped longitudinal frequency can be calculated by applying the free-end conditions, with the following result [11]:

$$
f_{\mathrm{d}, \mathrm{j}}=2 \pi \omega_{\mathrm{d}, \mathrm{j}}=\left[\frac{j^{2}}{4 L^{2}}\left(\frac{E_{\mathrm{H}}}{\rho}\right)-\frac{\gamma^{2}}{16 \pi^{2}}\right]^{1 / 2}
$$

where $L$ and $\rho$ are the length and density of the rod, and $j$ is an integer associated with each longitudinal mode.

Hence, the Young's modulus for any given applied magnetic field $E_{\mathrm{H}}$ can be estimated as a function of the measured first damped longitudinal frequency $f_{\mathrm{d}, 1}$ and its corresponding logarithmic decrement $\delta$ by the expression:

$$
E_{\mathrm{H}}=4 L^{2} \rho f_{\mathrm{d}, 1}^{2}\left(1+\frac{\delta^{2}}{4 \pi^{2}}\right)
$$

whereas the specific damping capacity for the same magnetic field $\Psi_{\mathrm{H}}$ deserves a further explanation.

Basically, two problems have been detected when measuring magnetomechanical damping by means of logarithmic decrement in free vibration:

(i) High stress-dependence of magnetomechanical damping

(ii) Measurement of an averaged logarithmic decrement along the full bar

The first problem is inherent to free decay measurement since the attenuation of oscillations leads to reduced stresses. Some works which are also based on free decay measurement, like torsional pendulum methods, have solved this problem by calculating the logarithmic decrement using a short portion of the signal [10]. Then, the estimated value can be associated to a particular applied stress.

The second problem is a bit more complex. Due to the fact that the logarithmic decrement is measured at the end section of the bar, the value obtained represents 
the averaged sum of every section of the bar, which may differ from the real value. Taking into account Lazan's work, mechanical losses can be written as an exponential stress-dependence [24]:

$$
\Delta W_{\mathrm{H}, \sigma}=J \sigma^{n}
$$

and the expression for the averaged specific damping capacity is given by:

$$
\left.\Psi_{\mathrm{H}, \sigma}\right|_{\mathrm{bar}}=\frac{\left.\Delta W_{\mathrm{H}, \sigma}\right|_{\mathrm{bar}}}{\left.W_{\mathrm{H}, \sigma}\right|_{\mathrm{bar}}}=\frac{\int_{0}^{L} J A \sigma^{n} \sin ^{n}\left(\frac{\pi x}{L}\right) \mathrm{dx}}{\int_{0}^{L} \frac{A \sigma^{2}}{2 E_{\mathrm{H}}} \sin ^{2}\left(\frac{\pi x}{L}\right) \mathrm{dx}}=\left.2 \delta_{\mathrm{H}, \sigma}\right|_{\mathrm{bar}}
$$

where $A$ is the cross section area of the bar and parameters $J$ and $n$ for ferromagnetic materials are not constant but depend on both applied stress and magnetic field [3]. So, the averaged estimation of $\left.\Psi_{\mathrm{H}, \sigma}\right|_{\text {bar }}$ (through $\left.\delta_{\mathrm{H}, \sigma}\right|_{\text {bar }}$ measurement) for different applied stresses and magnetic fields can be used to fit both $J$ and $n$, and then use Lazan's expression in order to obtain the desired $\Psi_{\mathrm{H}, \sigma}$ as follows:

$$
\Psi_{\mathrm{H}, \sigma}=\frac{\Delta W_{\mathrm{H}, \sigma}}{W_{\mathrm{H}, \sigma}}=\frac{J \sigma^{n}}{\frac{\sigma^{2}}{2 E_{\mathrm{H}}}}=2 E_{\mathrm{H}} J \sigma^{n-2}
$$

\section{Experimental set-up}

Figure 1 shows a general scheme of the experimental arrangement used in the present work. One may distinguish four parts constituting the full set-up. A PC equipped with a data acquisition system automatically controls the required procedure, which consists of magnetizing a ferromagnetic specimen, exciting it longitudinally, and finally measuring its vibration.

The ferromagnetic samples are magnetized by a solenoid in whose inner space the specimen is placed. In order to generate the magnetic field necessary to achieve the desired magnetization throughout the sample, a DC supply feeds the appropriate current intensity. The particular DC supply used is a Delta Elektronika SM400-AR-8 which provides $1600 \mathrm{~W}$, i.e., up to $4 \mathrm{~A}$ between $0 \mathrm{~V}$ and $400 \mathrm{~V}$, and is equipped with RS232 connectivity.

The magnetizing solenoid must be carefully designed in order to achieve the critical goals of sufficient magnetization capacity and great homogeneity of the applied magnetic field. These two requirements were met by combining two different contributions: a straight solenoid which gives high values of the magnetic field, and a pair of Helmholtz coils which compensate the inhomogeneity of the straight component. Some further important constraints had to be taken into account for a satisfactory design, because at maximum power of the DC supply the Joule effect losses can lead to a dangerously high temperature inside the coil. The final optimized design was a solenoid of $250 \mathrm{~mm}$ in length and $35 \mathrm{~mm}$ in inner diameter. As expected, the experimental results shown in Figure 2 for the magnetic field due to the Helmholtz coils, the straight solenoid, and both together confirmed that this last was the best configuration. The device can reach 
up to 2400 Oe, high enough to magnetize ferromagnetic materials such as nickel even when the demagnetizing field is considered [2, 22], and it provides a notable $140 \mathrm{~mm}$ band of homogeneity in which the magnetic field is within $2 \%$ of its central value. This characterization was carried out with a Walker Scientific MG-4D gaussmeter in vertical position and a micrometric table, and the measurements were normalized with regard to the vertical Earth's magnetic field. With respect to its thermodynamic response, the coil reaches nearly $50^{\circ} \mathrm{C}$ after working at maximum power for 5 minutes. This does not represent a real problem, however, because most trials are performed at considerably below maximum power; and since the increase in temperature is exponentially slower as the power decreases, the coil can be safely used for longer times.

This experimental set-up can be used to make measurements on ferromagnetic specimens of a wide range of shapes and sizes. In particular, it is possible to measure constant cross-section wires and bars up to $30 \mathrm{~mm}$ in diameter, with a limit of 140 $\mathrm{mm}$ length for the sample to be considered uniformly magnetized. Note that it is also unfeasible to use short specimen lengths due to the high demagnetizing field they generate [22], and due to their longitudinal resonance frequencies [23] being too high for the measurement devices. The set of pieces responsible for fixing the sample inside the solenoid consists of an opened ring to act as a clip, and a flanged cylinder to hold the ring in place inside the solenoid (Figure 3a). A screw joining the ring's opened surfaces is used to tighten it around the specimen rod, and rod and ring are inserted into the inner cylindrical space of the solenoid until they come to rest against a ledge machined into the inner surface of the solenoid at the appropriate position about halfway down. The flanged cylinder is then inserted into the solenoid on top of the ring and screwed into place in order to hold the ring in its correct position, avoiding its vertical displacement. Finally, the requirement of free longitudinal vibration requires locating the optimal position on the sample at which to hold it in the ring. For the first longitudinal mode, the vibrational node is at the centre of the rod, which is where the ring has to be placed in order to simulate the absence of constraints.

The excitation of the sample will also induce vibrations of the entire mechanical contraption used to hold the sample. Strictly speaking, the measured resonance frequency is not the actual longitudinal resonance frequency of the bar, but that of the entire mechanical system. Nevertheless, the influence of the mechanical fixture is negligible. In order to quantitatively prove this statement, the resonance frequencies of the entire mechanical system were compared to the resonance frequencies of bars tested with free boundary conditions (simulated by resting the rod on a little rubber block placed under its central zone). The averaged variation between the real free resonance and that measured with the mechanical contraption is low enough (less than $0.045 \%$ ) as to be within the limits of measuring uncertainty.

The excitation system is responsible for generating a free longitudinal oscillation in the specimen. The main characteristic required of this system is the ability to generate a suitable longitudinal excitation while avoiding or minimizing undesired modes such as torsion and bending. It should also permit the full control and automation of sequences 
of excitations.

A scheme of the excitation system used is shown in Figure 1. It consists of a barrel in which a lead pellet is placed, while a $2 / 2$ way valve and a relay regulate the necessary compressed air flow for the shot. The main dimensions and internal shape of the barrel are shown in Figure 3b. With the magnetizing solenoid placed vertically, the funnel-shaped end of the barrel collects the rebounding pellet and directs it downwards to the starting point under gravity. With this arrangement, there is full control and automation of the perpendicular impacts on the base of the specimen causing its vibration. Automation in a horizontal set-up would require the implementation of a complex mechanical magazine.

To obtain a useful longitudinal time-response, a calibration procedure was followed to optimize the triggering impact, determining the most appropriate values of the gap between the narrowest cross-section of the funnel and the base of the specimen rod, and of the range of compressed-air pressures. The final values were $30 \mathrm{~mm}$ for the gap providing the greatest number of successful impacts, and 3.5 to 4 bar for the range of shooting pressures.

The basis of the measuring system is a Polytec compact laser vibrometer. This consists of a CLV-1000 laser module and a CLV-700 sensor head. It is an example of a 'laser Doppler vibrometer', an optical instrument which employs laser technology to measure the out-of-plane velocity of single points of a vibrating object [15]. In the present device, a $70 \mathrm{MHz}$ He-Ne laser beam strikes a point on the vibrating surface and the light reflected travels back to the sensor head. The back-scattered light shows a Doppler-shifted frequency proportional to the vibration velocity.

This particular vibrometer can measure up to $1250 \mathrm{~mm} / \mathrm{s}$ in a bandwidth of 250 $\mathrm{MHz}$, which is broad enough to detect the frequencies expected according to the Nyquist theorem. The measurement resolution (the RMS signal amplitude at which the signalto-noise ratio is $0 \mathrm{~dB}$ in a $10 \mathrm{~Hz}$ spectral resolution) does not exceed $2 \mu \mathrm{m} / \mathrm{s}$ under any circumstances.

Input and output signals are handled with National Instruments acquisition devices which are controlled by a generic laptop. In this instance, we used a generic laptop connected to a series of National Instruments data acquisition and signal conditioning devices. In particular, these were a DAQCard $6062 \mathrm{E}$ with $500 \mathrm{kS} / \mathrm{s}$ and PCMCIA connectivity, and an SCC2345 multiplexer connecting the following modules: an SCCAO10 sending the necessary analog voltage to set the desired current in the DC supply, an SCC-RLY01 which functions as a relay controlling the compressed air shots, and an SCC-FT01 to receive the raw analog signal from the laser.

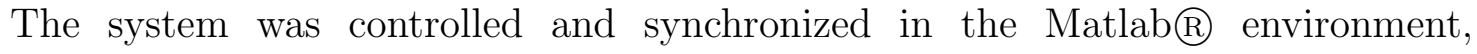
which, as well as being compatible with the National Instruments hardware, provides comprehensive data processing and decision making tools with a user-friendly graphical user interface. 


\section{Parameter estimation and data processing}

The data processing will be described next in detail in order to show how $E_{\mathrm{H}}$ and $\Psi_{\mathrm{H}, \sigma}$ are estimated.

(i) Data processing starts after storing the longitudinal vibration velocity timeresponse of a ferromagnetic rod through which a stress pulse is made to travel. Figure 4a shows an example of a signal acquired from the laser at a sampling rate of $100 \mathrm{kS} / \mathrm{s}$ for $0.1 \mathrm{~s}$ (resulting in a length of 10000 samples).

(ii) The next step is to use a frequency technique such as the Fast Fourier Transform (FFT) to determine the longitudinal vibration frequencies of the samples. A typical result of this process is shown in Figure 4b, where the first and second longitudinal modes clearly stand out.

(iii) Once the damped frequency corresponding to the first longitudinal mode has been successfully detected, one needs a filter to remove the contributions from undesired modes and noise. With this aim, several digital passband filters such as Butterworth, Bessel and Chebyshev were tested with admisible results, but a little noise contribution still remained on the signal. So, a novel non-parametric technique used in the analysis of time series and based on principles of multivariate statistics was used: the Singular Spectrum Analysis (SSA) [25]. The conditioned time-response signal which is obtain by means of SSA filtering is shown in Figure $4 c$.

(iv) This conditioned signal is finally subjected to a fitting procedure, not only to estimate an averaged attenuation constant $\frac{\gamma}{2}$ in accordance with equation (5) but also to ensure the quality of the acquired data and consequently the accuracy of the estimate. Two exponentials are fitted to the signal (Figure 4d), one to the positive part of the envelope, and another to the negative part. The fitting curve with the greater $R^{2}$ statistical parameter is chosen to be the better fit. The $R^{2}$ statistic also serves as a rejection criterion, with the acquisition being rejected if $R^{2}$ is less than $99.9 \%$.

(v) Once the best envelope has been chosen, the value of $E_{\mathrm{H}}$ is estimated through the averaged $\delta$ and $f_{\mathrm{d}, 1}$ as shown in expression (7).

(vi) Next, the stresses corresponding to the chosen envelope are estimated by the following expression (see [26]):

$$
\sigma_{\mathrm{H}}(z, t)=E_{\mathrm{H}} \frac{\partial u_{\mathrm{z}}(z, t)}{\partial z}
$$

in order to calculate $\left.\delta_{\mathrm{H}, \sigma}\right|_{\text {bar }}$ within intervals in which the attenuation is lower than $20 \%$ and the mean stress can be assumed constant as in Atalay's method [10].

(vii) Taking $\left.\delta_{\mathrm{H}, \sigma}\right|_{\text {bar }}$ for each mean stress, the losses per cycle can be estimated as follows:

$$
\left.\Delta W_{\mathrm{H}, \sigma}\right|_{\mathrm{bar}}=\left.\left.2 \delta_{\mathrm{H}, \sigma}\right|_{\mathrm{bar}} W_{\mathrm{H}, \sigma}\right|_{\mathrm{bar}}
$$


(viii) By linearly fitting the curve $\log \left(\left.\Delta W_{\mathrm{H}, \sigma}\right|_{\text {bar }}\right)$ vs. $\log (\sigma)$ (also shown in Figure $4 \mathrm{~d}$ ), the variable index $n$ can be estimated, while the variable $J$ can be determined from expression (9) as follows:

$$
J=\frac{\left.2 \delta_{\mathrm{H}, \sigma}\right|_{\text {bar }} \int_{0}^{L} \frac{A \sigma^{2}}{2 E_{\mathrm{H}}} \sin ^{2}\left(\frac{\pi x}{L}\right) \mathrm{dx}}{\int_{0}^{L} A \sigma^{n} \sin ^{n}\left(\frac{\pi x}{L}\right) \mathrm{dx}}=\frac{\left.\delta_{\mathrm{H}, \sigma}\right|_{\mathrm{bar}} L \sigma^{2-n}}{2 E_{\mathrm{H}} \int_{0}^{L} \sin ^{n}\left(\frac{\pi x}{L}\right) \mathrm{dx}}
$$

The integral must be computed numerically since the primitive only exists for positive natural values of index $n$.

(ix) Finally, the estimated $J$ and $n$ parameters of Lazan's expression allow us to calculate the real specific damping capacity at any given magnetic field and stress within our working interval thanks to equation (10).

The desired $\Delta E$ and $\Delta \Psi$ effects are easily estimated by repeating the previous data processing while the magnetic field is gradually increased.

\section{Experimental results}

The experimental set-up and procedure described in previous sections was tested by applying it to the magnetoelastic characterization of bulk ferromagnetic specimens represented by 9 nickel 201 rods of $10 \mathrm{~mm}$ diameter and $110 \mathrm{~mm}$ length. These dimensions were chosen so as to remain within the $2 \%$ band of uniform magnetic field. Metallographic analysis gave a purity of $(99.90 \pm 0.10) \%$ and a density of $8912 \mathrm{~kg} / \mathrm{m}^{3}$. The manufacturing process was cold rolled and the material was used as received from manufacturer (INCO Alloys International).

The experimental magnetic hysteresis loop of nickel, shown in Figure 5, was used to estimate useful magnetic magnitudes such as the coercive field $H_{\mathrm{c}}$ and the saturation magnetization $M_{\mathrm{s}}[2]$. The results are coherent with the typical shape of a magnetic hysteresis loop, with the following significant points being clearly identifiable: point (a) represents the demagnetized state, (b) and (c) the positive and negative magnetic retentivities after an irreversible magnetization, and (d) and (e) the positive and negative states of magnetic saturation.

Magnetic domain theory is necessary to understand the different mechanisms of magnetization and their relation to the measured results [27]. When low magnetic fields are applied, domain walls start to move in order to increase the size of those domains which have a net magnetization parallel to the external field lines. If applied magnetic field continues increasing, domain boundaries obtain enough energy to go through crystal imperfections or residual microstresses, which leads to irreversible displacements and the retentivities (b) or (c) when the applied field is removed. Finally, if the magnetic field becomes still more intense, magnetic domains will rotate in order to form a single domain, which means that the saturated points (d) or (e) are achieved, and the material behaves like a non-magnetic material.

Given the dimensions of these samples and the described magnetization curve of nickel, the demagnetizing effect cannot be neglected. This effect is due to the appearance 
of a pair of magnetic poles inside the ferromagnetic rod when an external magnetic field is applied. As a result, an internal demagnetizing field is created in the opposite direction to the applied field. The strength of this demagnetizing field is a function of both the magnetization state of the material and the geometrical dimensions of the sample [22]. The effective magnetic field $H_{\text {eff }}$ which really acts within the test specimen can be estimated by the expression:

$$
H_{\mathrm{eff}}=H_{\mathrm{s}}-H_{\mathrm{d}}=H_{\mathrm{s}}-N_{\mathrm{d}} M
$$

where $H_{\mathrm{s}}$ is the field generated by the solenoid, $H_{\mathrm{d}}$ the demagnetizing field, $N_{\mathrm{d}}$ the demagnetizing factor, and $M$ the magnetization of the material. The experimental magnetic hysteresis loop of nickel (Figure 5) was obtained and used to estimate the magnetization of the specimen, and the demagnetizing factor for a ferromagnetic rod can be calculated in terms of the ratio $r$ between its length and its diameter [22]. In the present case, the estimated saturation magnetization is $496 \mathrm{emu} / \mathrm{cm}^{3}$, and the demagnetizing factor is found to be $N_{\mathrm{d}}=0.2201$, which leads to a demagnetizing field at saturation equal to 109.2 Oe.

Clearly, the magnetization state of a ferromagnetic sample notably influences its behaviour because of the different magnetization curves that a specimen can follow [2]. In what follows, these curves will be described with reference to the points marked on Figure 5. In particular, there are three possibilities: magnetization from the demagnetized state (curve a-d), magnetization from a point of positive retentivity (curve b-d), and magnetization from a point of negative retentivity (curve c-d). Note that the first curve can only be followed the first time that the material is magnetized or after demagnetization, which can be achieved by heating the material to above its Curie temperature or by applying a high-frequency magnetic field to the specimen.

All three possibilities were considered in order to determine the influence of the sample's initial magnetization state on the $\Delta E$-effect and $\Delta \Psi$-effect results. In particular, each specimen was tested three times: the first for the a-d curve, the second for the b-d curve, and the third for the c-d curve. In each of these tests, a mesh of 45 different magnetic values was programmed, with much finer separation in the low magnetic field range where the main information is to be found. The test procedure was repeated 4 times at every point in order to attain sufficient statistical precision. By programming a waiting time of 2 seconds between consecutive triggers, and with a typical $80 \%$ success rate of excitation impacts, we were able to perform each test of 180 points in only 11 minutes. Figure 6 shows the $\Delta E$-effect and $\Delta \Psi$-effect curves obtained for the aforementioned three cases by statistically averaging the 9 samples tested.

With respect to the evolution of the Young's modulus shown in Figure 6a, two zones can be detected in the three cases studied: an initial stage of rapid growth which belongs to the low magnetic field range (less than $150 \mathrm{Oe}$ ), and a second stage of slow growth until saturation. These results agree with the magnetic domain theory. Low magnetic fields lead to easy displacements of domain walls whereas high ones imply the saturation of the sample in a single magnetic domain and the appearance of an upper limit which 
corresponds to the value of Young's modulus if the material were non-magnetic [1]. The main differences appear in the initial value of Young's modulus. Obviously, if the specimen has a retentivity due to a previous magnetization, the initial value of the Young's modulus will be greater, but after a process of domain reorganization [27] the three curves follow the same trend and reach the same value at saturation.

Again one observes two different zones in the specific damping capacity curve of Figure 6b: an initial rising stage which corresponds to the low applied magnetic field range (less than 150 Oe), and a second declining stage until saturation. This special trend can be again explained thanks to the magnetic domain structure of ferromagnetic materials. In the first stage of magnetization the damping increases as domain boundaries move irreversibly, whereas the second declining stage starts when the applied field is strong enough to suppress domain walls (by means of domain rotations) and make the specimen behave like a non-magnetic material [1]. The main differences are also attributable to the initial state of magnetic retentivity, since the initial value of the specific damping capacity will be greater when the specimen has been previously magnetized. Indeed, in this case the influence of the positive or negative retentivity is far more noticeable: when the specimen follows the c-d curve, the magnetic domains are mainly oriented initially in the direction opposite to the applied magnetic field in accordance with domain theory [27]. They should thus flip their orientation quickly as the field is applied, with the result that the curve has a local minimum which is rapidly rectified followed by convergence with the other curves.

Although the working stresses are much reduced, the proposed method is also able to study the stress-dependence of magnetomechanical damping in ferromagnetic materials at low stress levels. As it has been detailed in the explanation of data processing, two or more damping estimations are carried out for each measured signal. Figure 7 shows field-dependent specific damping capacity subjected to a stress of 1.0 $\mathrm{MPa}$ and $0.5 \mathrm{MPa}\left(\Psi_{1.0 \mathrm{MPa}}\right.$ and $\Psi_{0.5 \mathrm{MPa}}$, respectively), whose results are consistent with magnetomechanical damping theory $[1,3]$. Basically, magnetic domain motions are achieved by applying both magnetic fields and stresses, so stress-dependent curves will follow similar trends [3]. Our experimental system involves stress levels which are low enough not to saturate the samples, but they induce magnetic domain motions that increase the material damping while magnetization is lower than magnetic saturation. On the other hand, high magnetic fields make the sample saturate and become independent of stress because it behaves like a non-magnetic material.

Table 1 presents numerical results of interest for the $\Delta E$-effect. These lie within the range of values collected by Ledbetter and Reed [28], where the values of Young's modulus for demagnetized and saturated state for the more similar test conditions are $206 \mathrm{GPa}$ and $221 \mathrm{GPa}$, respectively. The differences with our values are lower than $2 \%$, which can be easily ascribed to the influence of little differences in heat treatment [3, 29], material composition [3], applied magnetic field or even experimental uncertainties. So, a better agreement should be expected when comparing results obtained using the same molten material and the same manufacturing process, like those reported in [11]. In fact, 
they showed a variation of elastic modulus with magnetic field (from demagnetized state up to saturated state, which corresponds to curve b-d in our Figure 5) of about 2.5\% (with extreme values of $213.0 \mathrm{GPa}$ and $218.4 \mathrm{GPa}$ ) which is in really good agreement with our results. The minute differences (lower than $0.3 \%$ in both states) may be caused by the use of a solenoid which is not compensated with Helmholtz coils and does not magnetize the sample uniformly along its full length. Furthermore, uncertainties in [11] are higher than ours (0.54 GPa vs. 0.17 GPa).

Table 2 shows the main numerical results for the $\Delta \Psi$-effect when the applied stress is $1.0 \mathrm{MPa}$ and $0.5 \mathrm{MPa}$. Although these stresses are much lower than those in [30] for a very similar nickel, our results seems to be clearly in accordance with Adams' estimations, which suggest a specific damping capacity around $0.5 \%$ at demagnetized state and less than 1.5\% when a magnetic field is applied. Trémolet de Lacheisserie [1] and Atalay [31] also reported magnetomechanical damping results using a torsional pendulum with high purity zone-melted nickel wires as well as with amorphous wires. These results provide support for the obtained trend in our damping curves, although the concrete results are not comparable. Unfortunately, the results in [11] (although obtained for the same material) are not suitable for comparison of damping since they just measured 5 points obtaining a mere global estimation of damping along the bar.

We finish this section with a brief analysis of uncertainties. The uncertainties shown in tables were calculated following ISO recommendations [32], with both type A and type B uncertainties taken into account. Type A uncertainty stems from the statistical averaging of the specimens and 4 replicates for each point characterized. Type B uncertainty is related to the equipment and material constants.

According to equation (7), Young's modulus estimates are affected by type B uncertainties related to density, geometrical dimensions, frequency, and logarithmic decrement, where the latter comprises the contributions from the laser Doppler vibrometer and the data acquisition equipment. The density uncertainty provided by the manufacturer was $8 \mathrm{~kg} / \mathrm{m}^{3}$; the manufacturing precision of the CNC turning centre used to cut the samples corresponded to a guaranteed maximum variation of $0.02 \mathrm{~mm}$; the sampling rate of the data acquisition hardware led to a value of $10 \mathrm{~Hz}$ for frequency resolution in the Fourier analysis; and laser resolution and target percent of reading were $0.5 \mu \mathrm{m} / \mathrm{s}$ and $0.0714 \%$, respectively.

Type B uncertainties have a similar influence in the determination of specific damping capacity since the Young's modulus is used to estimate it. In addition, the contribution of stress, index $n$ and constant $J$ must be taken into account according to equation (8). The former is considered a constant value in the range of $0.1 \mathrm{MPa}$, while $n$ and $J$ are defined by previously described contributions.

\section{Conclusions}

An improved method for the simultaneous measurement of the magnetoelastic $\Delta E$ and $\Delta \Psi$ effects has been applied to slender rods of crystalline nickel, also studying the 
influence of the initial magnetization state of the specimen on these effects.

The methodological approach was based on measuring the longitudinal component of the vibrational velocity of the specimen while an applied magnetic field magnetizes it from its demagnetized state up to its saturated state. The vibration was detected by a laser Doppler vibrometer, and excitation was by means of an automatically controlled compressed air system which repeatedly shoots a pellet against the sample.

The optimized characteristics of the experimental set-up endow the method with notable advantages over other techniques. First, the processes of excitation and of measurement do not affect the specimen's magnetization state. This thus increases the method's accuracy and allows measurement over the full range of magnetization. Second, the LDV technology has features which represent clear improvements in measurement (high accuracy, speed of measurement, and no need for sample preparation) over traditional or even ESPI sensors [15]. Third, stress-dependence of magnetomechanical damping can be studied and taken into account during data processing in order to avoid variations due to stress effects. And fourth, the automation and integration of the measurement and post-processing stages contributes to rapid, high resolution processing, making it possible to fully characterize (including post-processing) a mesh of more than 200 checked points in less than 15 minutes. In contrast, ESPI systems [11] can only measure (without post-processing) 8 non-checked points in more than one hour. Note that the difference between checked and non-checked points is that the former have passed a statistical rejection criterion which ensures their validity, whereas the latter have not.

Using this method, we obtained simultaneous high resolution curves of the magnetic field dependence of Young's modulus and specific damping capacity. This high resolution allowed us to observe effects that had been overlooked in previous works. First, a dependence of the $\Delta E$ and $\Delta \Psi$ effects on the initial magnetization state was found, which is consistent with the theory of magnetic domains and their irreversible movement [27]. Second, we detected a maximum value of the specific damping capacity just before the increment in Young's modulus starts to decline, which had also been shown by results based on torsional pendulum $[1,31]$, but that had gone undetected with lower resolution techniques as in [11].

\section{Acknowledgments}

This work was supported by the Consejería de Educación y Ciencia (Junta de Comunidades de Castilla - La Mancha, Spain) under Project PCI08-0082 "Análisis y diseño de elementos activos para el control de vibraciones". We also thank the Departamento de Física Aplicada of E.T.S.I. Industriales (UCLM) for their recommendations and help with the magnetic characterization of the nickel hysteresis loop, and Dr. F.J. Alonso for his SSA routines. The authors would also like to express their sincere gratitude to Dr. R.D. Adams for his advice and guidance in relation to magnetomechanical damping measurements. 


\section{References}

[1] du Trémolet de Lacheisserie E 1993 Magnetostriction: Theory and Applications of Magnetoelasticity (Boca Raton: CRC)

[2] Bozorth R M 1951 Ferromagnetism (New York: Van Nostrad Company Inc)

[3] Adams R D 1972 Damping of ferromagnetic materials at direct stress levels below the fatigue limit J. Phys. D: Appl. Phys. 5 1877-89

[4] Squire P T 1994 Magnetomechanical measurements of magnetically soft amorphous materials Meas. Sci. Technol. 5 67-81

[5] Hathaway K B and Spano M L 1984 Measurement of high magnetomechanical coupling factors by resonance techniques J. Appl. Phys. 55(6) 1765-7

[6] McSkimin H J 1964 Ultrasonic Methods for Measuring the Mechanical Properties of Liquids and Solids Physical Acoustics Vol 1A (New York: Academic Press)

[7] Malkinski L and Malkinski W 1990 Electronic system for the measurement of elasticity moduli and internal friction of thin metallic ribbons Meas. Sci. Technol. $1277-81$

[8] Chicharro J M, Bayón A and Salazar F 1999 Measurement of field-dependence elastic modulus and magnetomechanical coupling factor by optical heterodyne interferometry J. Magn. Magn. Mater. 202 465-72

[9] Nowick A S and Berry B S 1992 An Elastic Relaxation in Crystalline Solids (New York: Academic Press)

[10] Atalay S and Squire P T 1992 Torsional pendulum system for measuring the shear modulus and internal friction of magnetoelastic amorphous wires Meas. Sci. Technol. 3 735-9

[11] Chicharro J M, Bayón A and Salazar F 2004 Measurement of damping in magnetic materials by optical heterodyne interferometry J. Magn. Magn. Mater. 268 348-56

[12] Sumner G and Entwistle K M 1958 The measurement of strain-dependent damping of metals vibrating torsionally Brit. J. Appl. Phys. 9 434-8

[13] Adams R D and Percival A L 1969 Measurement of the strain-dependent damping of metals in axial vibration J. Phys. D: Appl. Phys. 2 1693-704

[14] Adams R D and Fox M A O 1972 Measurement of the damping capacity and dynamic modulus of high-damping metals under direct cyclic stresses J. Phys. D: Appl. Phys. 5 1274-83

[15] Castellini P, Martarelli M and Tomasini E P 2006 Laser Doppler Vibrometry: Development of advanced solutions answering to technology's needs Mechanical Systems and Signal Processing $201265-85$

[16] Gren P, Tatar K, Granström J, Molin N E and Jansson E V 2006 Laser vibrometry measurements of vibration and sound fields of a bowed violin Meas. Sci. Technol. 17 635-44

[17] Olsson E and Tatar K 2006 Sound field determination and projection effects using laser vibrometry Meas. Sci. Technol. 17 2843-51

[18] Staszewski W J, Lee B C and Traynor R 2007 Fatigue crack detection in metallic structures with Lamb waves and 3D laser vibrometry Meas. Sci. Technol. 18 727-39

[19] Salmi A, Karppinen T and Hæggström 2006 Measuring in-plane mechanical properties of plate-like samples using phonographic pickups Meas. Sci. Technol. 17 N97-N101

[20] Gregori G, Lì L, Nychka J A and Clarke D R 2007 Vibration damping of superalloys and thermal barrier coatings at high-temperatures Mater. Sci. Technol. Eng. A. 466 256-64

[21] Morales A L, Nieto A J, Moreno R, González A, Chicharro J M and Pintado P 2007 Simultaneous measurement of Young's modulus and damping dependence on magnetic fields by laser interferometry Proc. 8th. Int. Conf. on Thermal, Mechanical and Multi-Physics Simulation Experiments in Microelectronics and Micro-Systems (London) (Maastricht: Shaker Publishing / IEEE)

[22] Cullity B D 1972 Introduction to Magnetic Materials (Massachusetts: Addison-Wesley Publishing Company)

[23] Seto W 1970 Vibraciones Mecánicas (New York: McGraw-Hill) 
[24] Lazan B J 1960 Structural damping (Oxford: Pergamon Press)

[25] Golyandina N, Nekrutkin V and Zhigljavsky A 2001 Analysis of Time Series Structure: SSA and Related Techniques (Boca Raton: Chapman \& Hall / CRC)

[26] Timoshenko S and Young D H 1968 Elements of Strength of Materials (New York: Van Nostrad Company Inc.)

[27] Chikazumi S 1964 Physics of Magnetism (New York: John Wiley \& Sons, Inc.)

[28] Ledbetter H M and Reed R P 1973 Elastic properties of metals and alloys, I. Iron, Nickel and Iron-Nickel alloys Journal of Physical and Chemical Reference Data 2(3) 531-617

[29] Chicharro J M, Bayón A and Salazar F 2006 Dependence of $\Delta E$ effect on internal stresses in nickel: Experimental results by laser interferometry J. Magn. Magn. Mater. 297 44-53

[30] Adams R D 1967 The damping characteristics of certain steels, cast irons and other metals (DP Thesis, Cambridge University)

[31] Atalay S and Squire P T 1991 Field-dependent shear modulus and internal friction in annealed iron-based amorphous wires J. Magn. Magn. Mater. 101 47-8

[32] ISO (International Organization for Standardization) 1995 Guide to the Expression of Uncertainty in Measurement (Switzerland: ISO) 


\section{Figure captions}

Figure 1. Experimental set-up.

Figure 2. Experimental characterization of the magnetic field applied by the Helmholtz coils (light grey), the straight solenoid (grey), and both together (dark grey), depending on length and intensity.

Figure 3. Main dimensions of holder (a) and exciter (b).

Figure 4. Data processing: (a) raw acquired time-response, (b) FFT, (c) filtered timeresponse, and (d) curve fitting process and stress-dependence study.

Figure 5. Experimental magnetic hysteresis loop for the nickel specimens studied.

Figure 6. Results for the (a) $\Delta E$-effect and (b) $\Delta \Psi$-effect corresponding to an applied stress of 1.0 $\mathrm{MPa}$ for the first magnetization curve (solid line), the magnetization curve with positive retentivity (dashed line), and the magnetization curve with negative retentivity (dotted line).

Figure 7 . Results for the $\Delta \Psi$-effect for the magnetization curve with positive retentivity (a) and negative retentivity (b) corresponding to an applied stress of 1.0 MPa (solid line) and 0.5 $\mathrm{MPa}$ (dashed line).

\section{Tables}

Table 1. $\Delta E$-effect results (D-demagnetized, S-saturated).

Table 2. $\Delta \Psi$-effect results corresponding to applied stresses of $1.0 \mathrm{MPa}$ and $0.5 \mathrm{MPa}$ (D-demagnetized, GM-global maximum, S-saturated). 


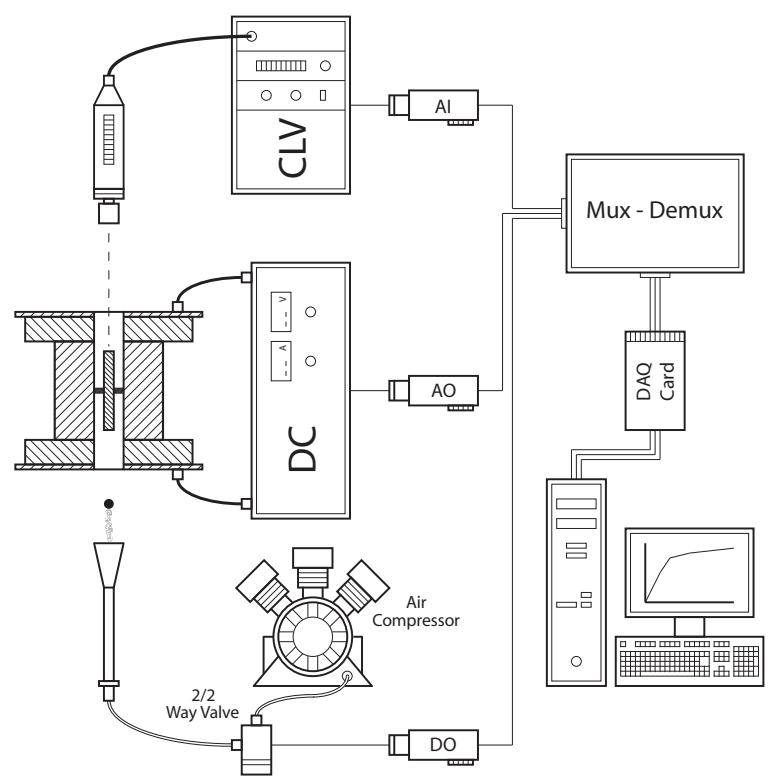

Figure 1. Experimental set-up 


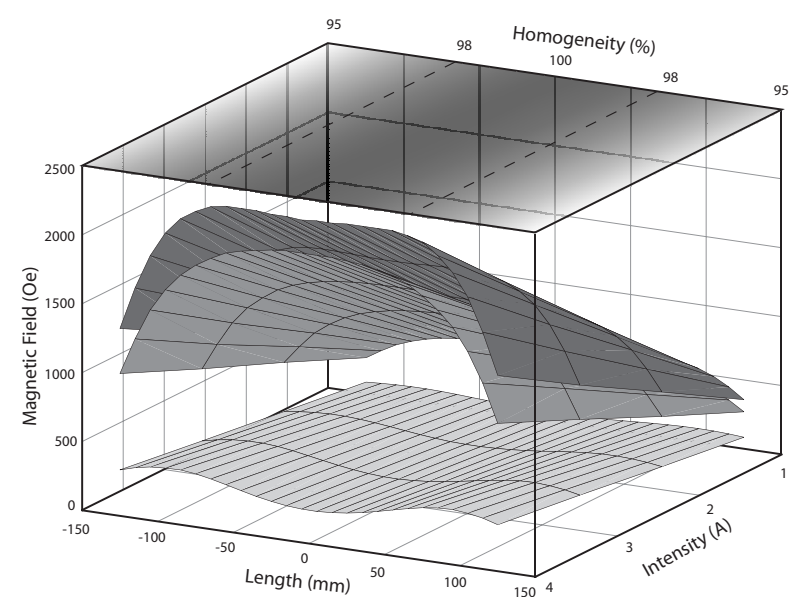

Figure 2. Experimental characterization of the magnetic field applied by the Helmholtz coils (light grey), the straight solenoid (grey), and both together (dark grey), depending on length and intensity 


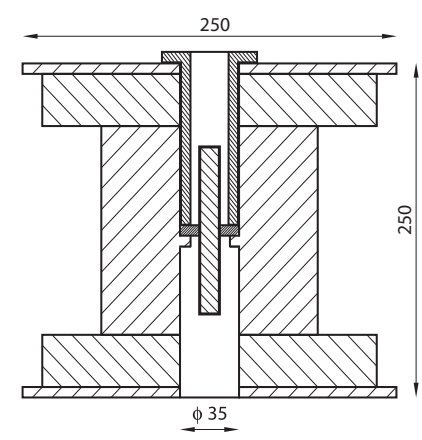

(a)

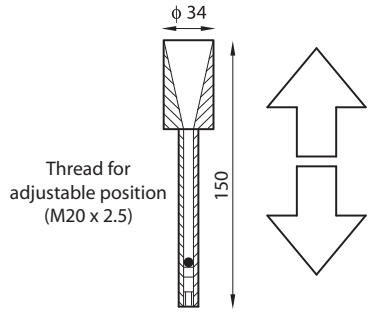

(b)

Figure 3. Main dimensions of holder (a) and exciter (b) 
(a)

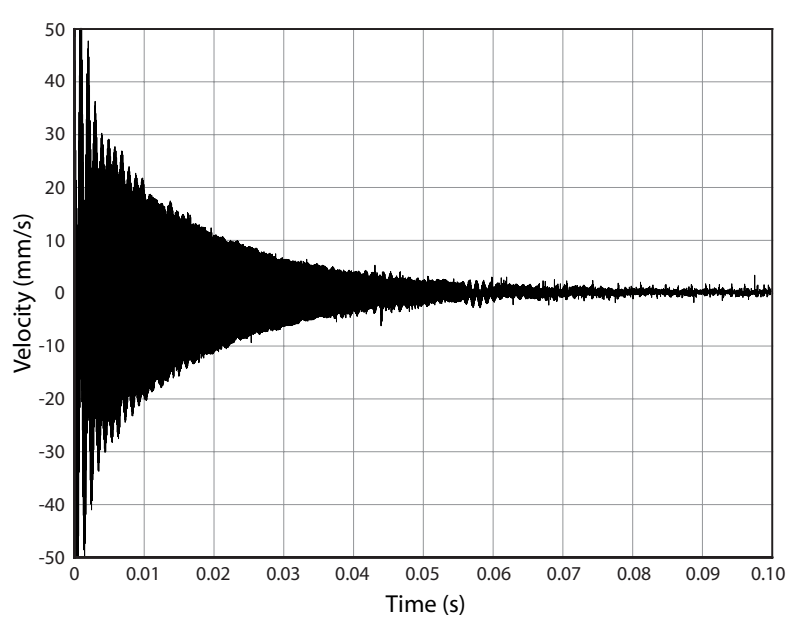

(c)

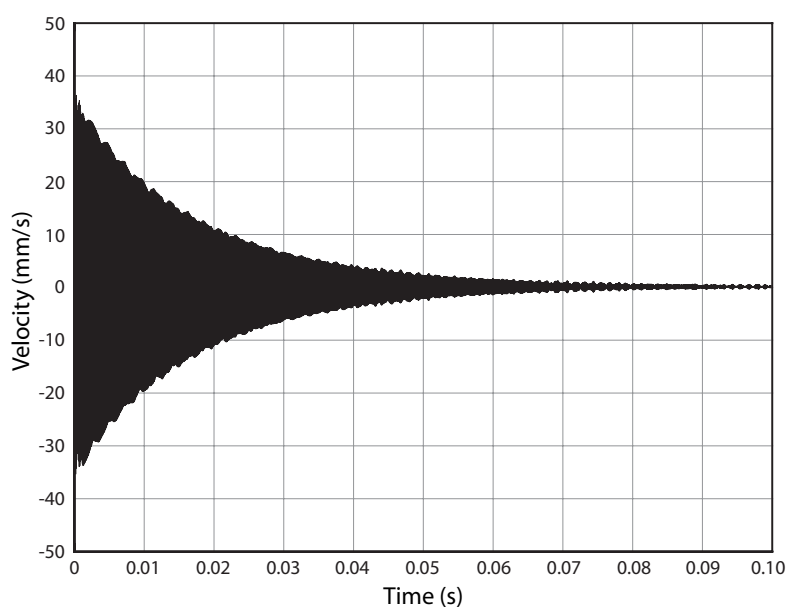

(b)

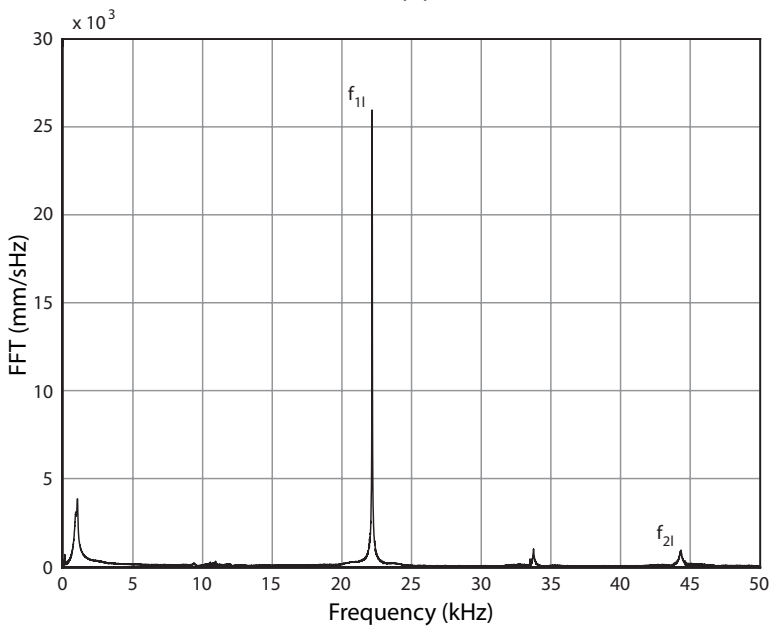

(d)

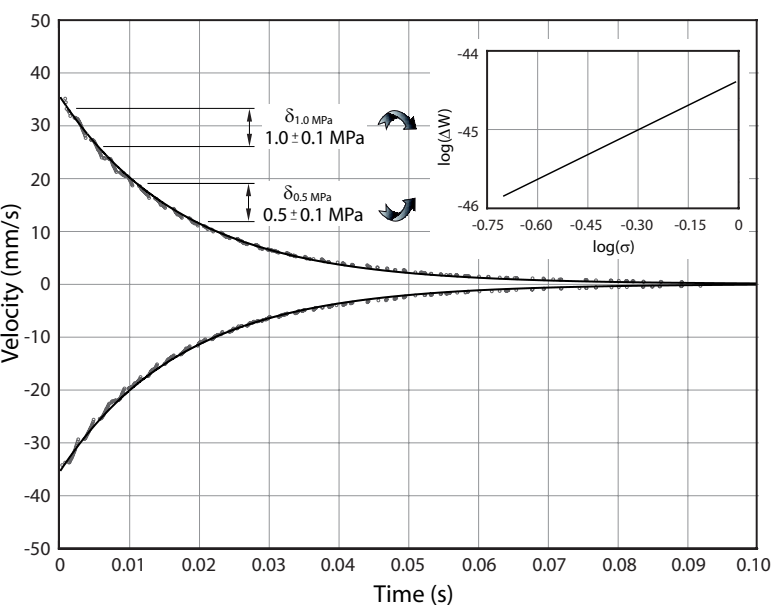

Figure 4. Data processing: (a) raw acquired time-response, (b) FFT, (c) filtered time-response, and (d) curve fitting process and stress-dependence study 


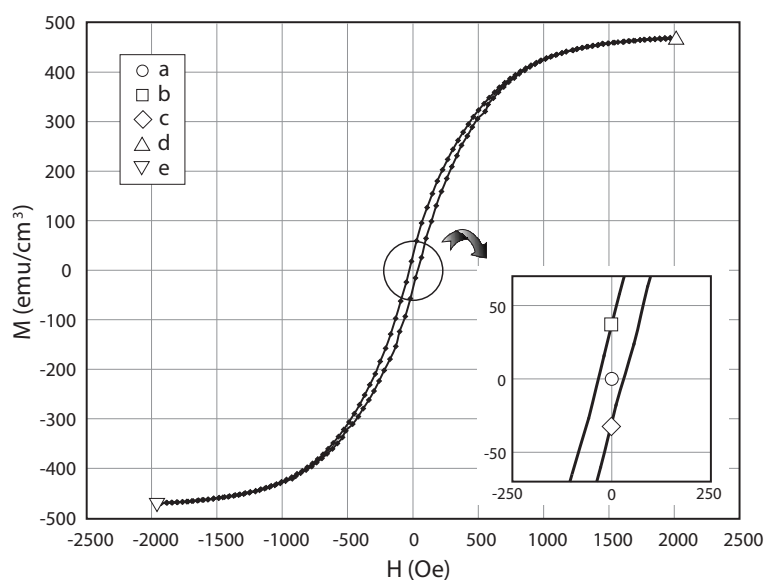

Figure 5. Experimental magnetic hysteresis loop for the nickel specimens studied 
(a)

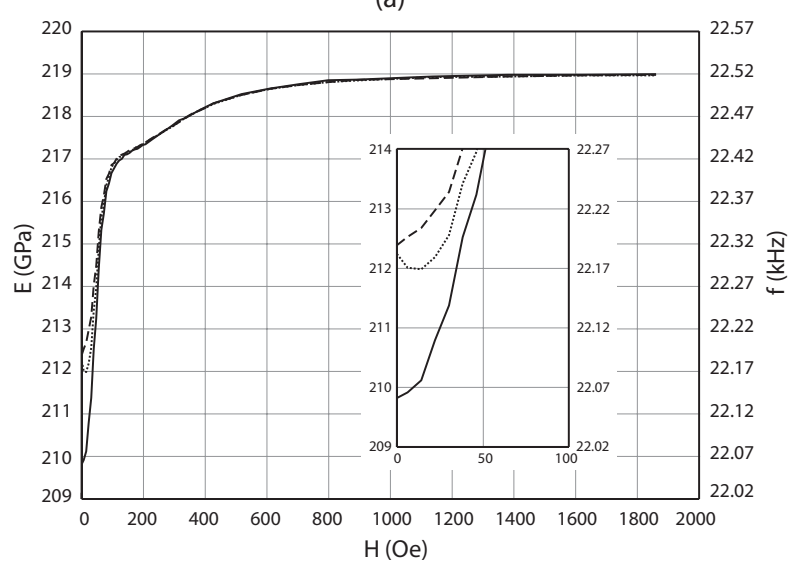

(b)

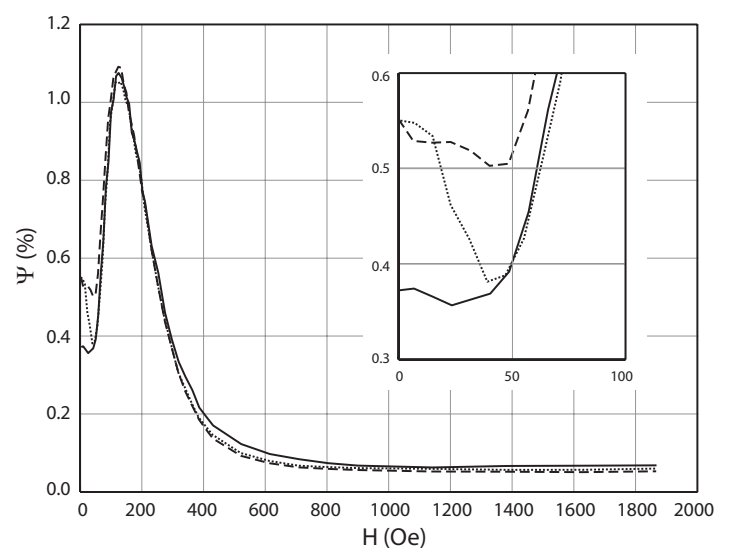

Figure 6. Results for the (a) $\Delta E$-effect and (b) $\Delta \Psi$-effect corresponding to an applied stress of 1.0 MPa for the first magnetization curve (solid line), the magnetization curve with positive retentivity (dashed line), and the magnetization curve with negative retentivity (dotted line) 
(a)

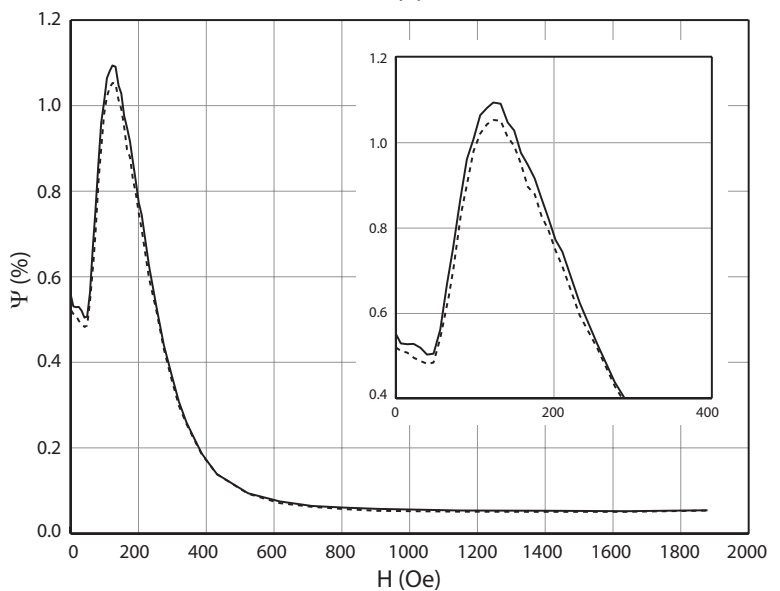

(b)

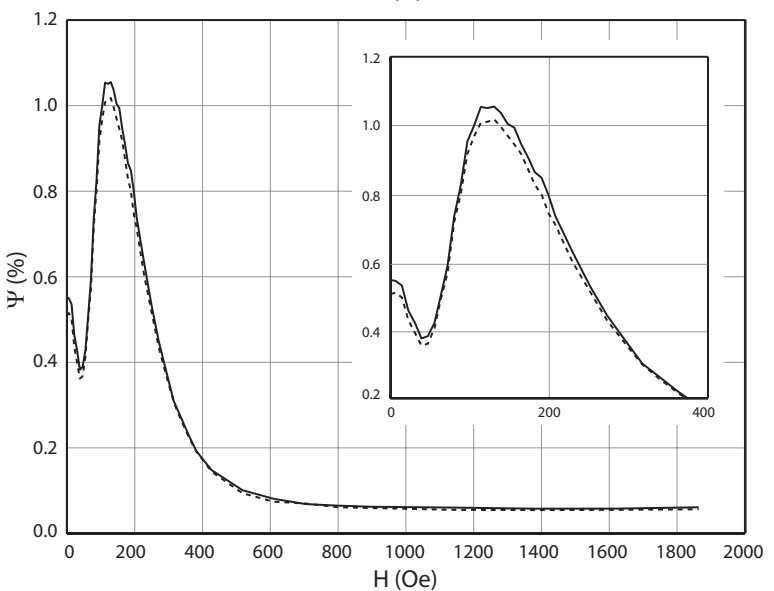

Figure 7. Results for the $\Delta \Psi$-effect for the magnetization curve with positive retentivity (a) and negative retentivity (b) corresponding to an applied stress of 1.0 $\mathrm{MPa}$ (solid line) and 0.5 MPa (dashed line) 
Table 1. $\Delta E$-effect results (D-demagnetized, S-saturated)

\begin{tabular}{|c|c|c|c|c|c|}
\hline Magnetization curve & State & $f_{1 l} \pm u\left(f_{1 l}\right)(\mathrm{Hz})$ & $E \pm u(E)(\mathrm{GPa})$ & $\Delta f_{1 l}(\mathrm{~Hz})$ & $\Delta E(\mathrm{GPa})$ \\
\hline \multirow{2}{*}{$\mathrm{a}-\mathrm{d}$} & $\mathrm{D}$ & $22055.36 \pm 5.77$ & $209.82 \pm 0.17$ & 476.98 & 9.17 \\
& $\mathrm{~S}$ & $22532.34 \pm 5.77$ & $218.99 \pm 0.17$ & $(2.16 \%)$ & $(4.37 \%)$ \\
\hline \multirow{2}{*}{ b-d } & $\mathrm{D}$ & $22190.01 \pm 5.77$ & $212.39 \pm 0.17$ & 341.50 & 6.59 \\
& $\mathrm{~S}$ & $22531.51 \pm 5.77$ & $218.98 \pm 0.17$ & $(1.54 \%)$ & $(3.10 \%)$ \\
\hline \multirow{2}{*}{ c-d } & $\mathrm{D}$ & $22182.38 \pm 5.77$ & $212.24 \pm 0.17$ & 348.45 & 6.72 \\
& $\mathrm{~S}$ & $22530.83 \pm 5.77$ & $218.97 \pm 0.17$ & $(1.57 \%)$ & $(3.17 \%)$ \\
\hline
\end{tabular}


Table 2. $\Delta \Psi$-effect results corresponding to applied stresses of $1.0 \mathrm{MPa}$ and $0.5 \mathrm{MPa}$ (D-demagnetized, GM-global maximum, S-saturated)

\begin{tabular}{|c|c|c|c|}
\hline Magnetization curve & State & $\Psi_{1.0 \mathrm{MPa}} \pm u\left(\Psi_{1.0 \mathrm{MPa}}\right)(\%)$ & $\Psi_{0.5 \mathrm{MPa}} \pm u\left(\Psi_{0.5 \mathrm{MPa}}\right)(\%)$ \\
\hline \multirow{3}{*}{$\mathrm{a}-\mathrm{d}$} & $\mathrm{D}$ & $0.371 \pm 0.033$ & $0.300 \pm 0.024$ \\
\cline { 2 - 4 } & $\mathrm{GM}$ & $1.073 \pm 0.089$ & $1.012 \pm 0.076$ \\
\cline { 2 - 4 } & $\mathrm{S}$ & $0.080 \pm 0.007$ & $0.074 \pm 0.007$ \\
\hline \multirow{3}{*}{ b-d } & $\mathrm{D}$ & $0.557 \pm 0.048$ & $0.525 \pm 0.040$ \\
\cline { 2 - 4 } & $\mathrm{GM}$ & $1.100 \pm 0.080$ & $1.056 \pm 0.075$ \\
\cline { 2 - 4 } & $\mathrm{S}$ & $0.058 \pm 0.005$ & $0.058 \pm 0.005$ \\
\hline \multirow{3}{*}{$\mathrm{c}-\mathrm{d}$} & $\mathrm{D}$ & $0.551 \pm 0.044$ & $0.510 \pm 0.039$ \\
\cline { 2 - 4 } & $\mathrm{GM}$ & $1.055 \pm 0.083$ & $0.054 \pm 0.005$ \\
\cline { 2 - 4 } & $\mathrm{S}$ & $0.059 \pm 0.005$ & \\
\hline
\end{tabular}

